\title{
Advanced magnetic anisotropy determination through isothermal remanent magnetization of nanoparticles
}

\author{
A. Hillion, ${ }^{1}$ A. Tamion, ${ }^{1,}$ F. Tournus, ${ }^{1}$ O. Gaier, ${ }^{2}$ E. Bonet, ${ }^{2}$ C. Albin,,${ }^{1}$ and V. Dupuis ${ }^{1}$ \\ ${ }^{1}$ Institut Lumière Matière, UMR5306 Université Lyon 1-CNRS, Université de Lyon 69622 Villeurbanne cedex, France \\ ${ }^{2}$ Institut Néel, CNRS-Université Joseph Fourier, BP 166, 38042 Grenoble Cedex 9, France \\ (Received 30 April 2013; revised manuscript received 22 July 2013; published 13 September 2013)
}

\begin{abstract}
We propose a theoretical framework enabling the simulation of isothermal remanence magnetization (IRM) curves, based on the Stoner-Wohlfarth model combined with the Néel macrospin relaxation time description. We show how low temperature IRM curves, which have many advantages compared to hysteresis loops, can be efficiently computed for realistic assemblies of magnetic particles with both a size and anisotropy constant distribution, and a biaxial anisotropy. The IRM curves, which probe the irreversible switching provoked by an applied field, are shown to be complementary to other usual measurements (in particular low-field susceptibility curves where a thermal switching is involved). As an application, the experimental IRM curve of Co clusters embedded in a carbon matrix is analyzed. We demonstrate how powerful such an analysis can be, which in the present case allows us to put into evidence an anisotropy constant dispersion among the Co nanoparticles.
\end{abstract}

DOI: 10.1103/PhysRevB.88.094419

PACS number(s): 75.75.-c, 75.20.-g, 75.30.Gw, 75.50.Tt

Magnetic nanoparticles are of great interest in a wide range of disciplines, including magnetic fluids, catalysis, biotechnology/biomedicine, magnetic resonance imaging, data storage, and environmental remediation. ${ }^{1-13}$ Successful applications of such magnetic nanoparticles in the areas listed above are highly dependent on the stability of the particles under a range of different conditions. In particular, these nanomagnets might be used as magnetic media in future high-density magnetic storage devices with ultimate recording bits, i.e., a single nanoparticle or even a single atom.${ }^{14}$ Reading and writing of such a system requires knowing perfectly its magnetic properties, especially its anisotropy constant.

Magnetic measurements on assemblies of well defined nanoparticles can be used to infer their intrinsic magnetic properties. Typically, zero field-cooled/field-cooled (ZFC/FC) susceptibility curves give information on both the magnetic size distribution and the effective anisotropy constant $K_{\text {eff }}{ }^{15-17}$ This technique, based on thermal switching of the nanomagnets, is indeed sensitive to the macrospin switching energy barrier, i.e., the magnetic anisotropy energy (MAE) $E_{\text {ani }}=K_{\text {eff }} V$, where $V$ is the particle volume. Therefore, even if it is a powerful method, it does not enable us to get a complete picture of the anisotropy energy landscape (components beyond the uniaxial anisotropy). On the other hand, low temperature hysteresis loops bear the signature of the different magnetic anisotropy contributions. ${ }^{18}$ However, in addition to analysis difficulties (i.e., extract the relevant signal from the spurious contributions like paramagnetic impurities, diamagnetic substrate...), they are difficult to model, ${ }^{19,20}$ often at the price of expensive numerical calculations: There is unfortunately no analytical description of hysteresis loops.

In this paper, we propose a convenient method which obliterates these difficulties, while enabling at the same time an accurate depiction of the magnetic anisotropy. We show how an advanced magnetic anisotropy determination is possible through isothermal remanent magnetization (IRM) of nanoparticles.

Let us remind the reader the principle of this measurement. First, the nanoparticle sample has to be demagnetized by zero field cooling from the superparamagnetic (SP) state. Then, a magnetic field $H$ is applied before going back to zero: We then acquire the isothermal remanent magnetic moment. By successively increasing $H$, we finally obtain a full IRM $(H)$ curve. This curve necessarily starts from 0 (for $H=0$ ) and goes up to a maximum value which appears to be simply the remanent moment $m_{R}$ obtained after saturation (i.e., the same remanent state as for the major hysteresis loop). This protocol was initially used to evaluate the switching field distribution or, combined with direct current demagnetization (DcD) measurements, to detect the nature of interactions via the parameter $\Delta m=\operatorname{DcD}(H)-\left[m_{R}-2 \operatorname{IRM}(H)\right]$ or via a Henkel plot. ${ }^{21-30}$

IRM curves only reflect the irreversible switching of magnetic nanoparticles, and the signal (always measured at zero field) is free of any other magnetic contributions. Surprisingly, IRM curves seem to be often underrated and are rarely quantitatively analyzed. ${ }^{31,32}$ Here, we set up a simple IRM modeling based on the Stoner-Wohlfarth (SW) model and the Néel description for the macrospin switching time $\tau=\tau_{0} e^{\Delta E /\left(k_{B} T\right)}, \Delta E$ being the switching energy barrier. For a uniaxial magnetic anisotropy an analytical expression will be used, whereas for the more general case a numerical approach will be followed. This deterministic framework (as opposed to Monte-Carlo methods) allows us to describe IRM curves of nanoparticle assemblies with realistic parameters, namely taking into account the temperature, the particle size distribution, a $K_{\text {eff }}$ dispersion, a biaxial anisotropy, etc. The IRM protocol, where the magnetization switching is due to an applied field, appears to be complementary to ZFC/FC susceptibility where the external field is close to zero and the switching is thermally activated. Combining these two magnetic measurements leads to an improved characterization of the nanomagnets intrinsic properties, as we will demonstrate on a diluted Co nanoparticle assembly. Results will be obtained, in particular a quantification of the anisotropy constant dispersion. Finally, we present the good qualitative agreement between $\mu$-SQUID experiments, on single Co nanoparticles, and SQUID measurements on assemblies of the same nanomagnets. 
Let us examine, for a single particle size and anisotropy energy, how the IRM $(H)$ curve can be modeled. In the following, we assume that, within the assembly, the noninteracting magnetic clusters are randomly oriented and undergo coherent magnetization reversal (macrospin approximation). In order to take into account the temperature, which enables the macrospin switching over $\Delta E$, we will assume that the reversal occurs when $\tau \leqslant \tau_{m}$, where $\tau_{m}$ is the measurement time (typically a few seconds). This condition is met for $\Delta E \leqslant \ln \left(\tau_{m} / \tau_{0}\right) k_{B} T \simeq 25 k_{B} T$. We now consider a uniaxial anisotropy, with a magnetic anisotropy constant $K_{\text {eff }}$. To a very good approximation, the evolution of the switching energy barrier can be written: $\Delta E(H)=K_{\mathrm{eff}} V\left[1-H / H_{\mathrm{sw}}^{0}(\theta)\right]^{3 / 2}$, where $H_{\mathrm{sw}}^{0}(\theta)$ is the switching field at zero temperature for nanomagnets with an angle $\theta$ between their easy axis and the applied field. Then, for each orientation, it is possible to express the switching field $H_{\mathrm{sw}}(\theta, T)$, for a given temperature: $H_{\mathrm{sw}}(\theta, T)=c H_{\mathrm{sw}}^{0}(\theta)$ with $c=1-\left[25 k_{B} T /\left(K_{\text {eff }} V\right)\right]^{2 / 3}$. The effect of temperature is a simple reduction, by a factor called here $c$, of the zero-temperature switching field. This field $H_{\mathrm{sw}}^{0}(\theta)$ varies between $H_{A} / 2$ and $H_{A}$, where the anisotropy field is defined by $H_{A}=2 K_{\text {eff }} /\left(\mu_{0} M_{S}\right), M_{S}$ being the particle saturation magnetization. Consequently, by introducing the reduced field $h=H /\left(c H_{A}\right)$, all the particles will switch between $h=0.5$ and $h=1$. We thus have for the assembly $\operatorname{IRM}(h<0.5)=0$ and $\operatorname{IRM}(h>1)=m_{R}$. In the interval $h \in[0.5,1]$, we can easily establish an analytical formula for $\operatorname{IRM}(H, T)$ :

$$
\begin{aligned}
\operatorname{IRM}(H, T) & =m_{R} \frac{1-x^{3}}{1+x^{3}} \text { with } \\
x & =\frac{1+2 h^{2}-\sqrt{12 h^{2}-3}}{2\left(1-h^{2}\right)}
\end{aligned}
$$

For a realistic nanomagnet assembly, there is necessarily a particle size distribution $\rho(V)$. The resulting IRM curve can then be calculated by

$$
\operatorname{IRM}(H, T)=\frac{M_{S}}{2} \int_{V_{\min }}^{\infty} \frac{1-x^{3}}{1+x^{3}} V \rho(V) \mathrm{d} V,
$$

which can be numerically evaluated without any difficulty. Here $V_{\min }=25 k_{B} T / K_{\text {eff }}$ is the volume under which the particles are superparamagnetic, and thus do not contribute to the IRM signal. In the above expression, the variable $x$ depends on the particle volume $V$, as well as on $H$ and $T$, and $\rho(V) \mathrm{d} V$ represents the number of particles having a volume in the interval $[V, V+\mathrm{d} V]$. Note that, with this analytical formulation, it is also straightforward to take into account an anisotropy constant distribution $f\left(K_{\text {eff }}\right)$. In such a case, all the nanomagnets of the same volume $V$ do not display the same anisotropy energy $E_{\text {ani: }}$ : They have a probability $f\left(K_{\text {eff }}\right) \mathrm{d} K_{\text {eff }}$ to have an anisotropy constant in the range [ $\left.K_{\text {eff }}, K_{\text {eff }}+\mathrm{d} K_{\text {eff }}\right]$.

Furthermore, it is possible to go beyond the usual uniaxial anisotropy approximation. Indeed, it has been shown using a $\mu$-SQUID study that Co clusters, in the fcc phase, have a biaxial anisotropy. ${ }^{33-36}$ This can be explained by the physical origin of the magnetic anisotropy which results both from the particle surface and its shape. Namely, particles deviating from a perfectly regular nanocrystal shape (truncated octahedron in the case of fcc $\mathrm{Co}$ ) will display, in the general case, a biaxial anisotropy. The anisotropy energy can then be expressed as $E_{\text {ani }} / V=K_{1} m_{z}^{2}+K_{2} m_{y}^{2}$, with $z$ the easy axis, $y$ the hard axis, $x$ the intermediate axis, $\mathbf{m}=\left(m_{x}, m_{y}, m_{z}\right)$ the unit vector directed along the particle magnetic moment, and $K_{1}<0<$ $K_{2}$. In this case there is no analytical expression of the astroid surface in the field space. Nevertheless, an IRM curve can be computed by a numerical approach. As for the uniaxial case, we start by considering the effect of temperature through a simple reduction of the zero-temperature switching field (which here depends on both angles $\theta$ and $\varphi$ defined by $\cos \theta=$ $H_{z} / H$ and $\left.\sin \theta \sin \varphi=H_{y} / H\right): H_{\mathrm{sw}}(\theta, \varphi, T)=c H_{\mathrm{sw}}^{0}(\theta, \varphi)$. In order to determine $H_{\mathrm{sw}}^{0}(\theta, \varphi)$, i.e., to build the astroid, we use the geometrical approach. ${ }^{33,37}$ In contrast to the uniaxial case, where the averaging over the macrospin orientations is performed analytically, followed by the averaging over the different particle sizes, we here fix an orientation $(\theta, \varphi)$ and determine a switching volume $V_{\mathrm{sw}}^{\theta, \varphi}(H, T)$. This volume is the maximum size verifying $H_{\mathrm{sw}}(\theta, \varphi, T) \leqslant H$ : It means that, with this particular orientation, all particles of volume $V \leqslant V_{\mathrm{sw}}^{\theta, \varphi}(H, T)$ will switch. A numerical integration can then be performed, first over the particle volume up to $V_{\mathrm{sw}}^{\theta, \varphi}(H, T)$, and next over the angles $\theta$ and $\varphi$ [which corresponds to a probability density function $\rho(\theta, \varphi)=\sin \theta / 2$ ] in order to determine the contribution of macrospins that have switched. In the end, we can write

$$
\mathrm{IRM}=2 \iint_{\theta, \varphi} \int_{V_{\min }}^{V_{\mathrm{sw}}^{\theta, \varphi}} M_{S} V \cos \theta \rho(V) \mathrm{d} V \rho(\theta, \varphi) \mathrm{d} \theta \mathrm{d} \varphi,
$$

where the dependence on $H$ and $T$ (and $K_{\text {eff }}$, if an anisotropy constant dispersion needs to be considered) is incorporated in the switching volume $V_{\mathrm{sw}}^{\theta, \varphi}(H, T)$. Note that, as expected the IRM goes from zero (at $H=0$ ) up to the remanent moment $m_{R}$ for a large applied field (when the switching volume goes to $\infty$ ).

Now that we have established expressions allowing us to efficiently simulate $\operatorname{IRM}(H)$ curves at a given temperature, this framework can be used to analyze experimental curves combined with other complementary measurements, namely ZFC/FC susceptibility curves and room temperature $m(H)$ superparamagnetic loops for which a theoretical description is also available. We consider here a benchmark sample of $\mathrm{Co}$ nanoparticles, with a mean diameter around $2.5 \mathrm{~nm}$ (lognormal size distribution, with a typical standard deviation of $0.7 \mathrm{~nm}$ ), prepared in the gas phase and deposited fragmentation free onto a $\mathrm{Si}$ substrate together with an amorphous carbon matrix. ${ }^{15,18,38,39}$ It has been shown that the Co particles are well crystalized in the fcc phase, with truncated octahedral shapes. In this case, the biaxial magnetic anisotropy comes from the finite particle size and is controlled by the facetting. ${ }^{34,36}$

Given the very low Co concentration $(0.5$ vol. \%, which corresponds to a mean interparticle distance around $8 \mathrm{~nm}$ ), we expect the interparticle interactions to be negligible. In order to verify this assumption, we take advantage of the IRM and DcD measurements. For such a diluted sample, as shown in Fig. 1(b), the $\Delta m$ parameter calculated from IRM and DcD curves (respectively, dots and squares) is equal to zero within the uncertainty, whatever the applied magnetic field: There is thus no detectable magnetizing $(\Delta m>0)$ or demagnetizing 


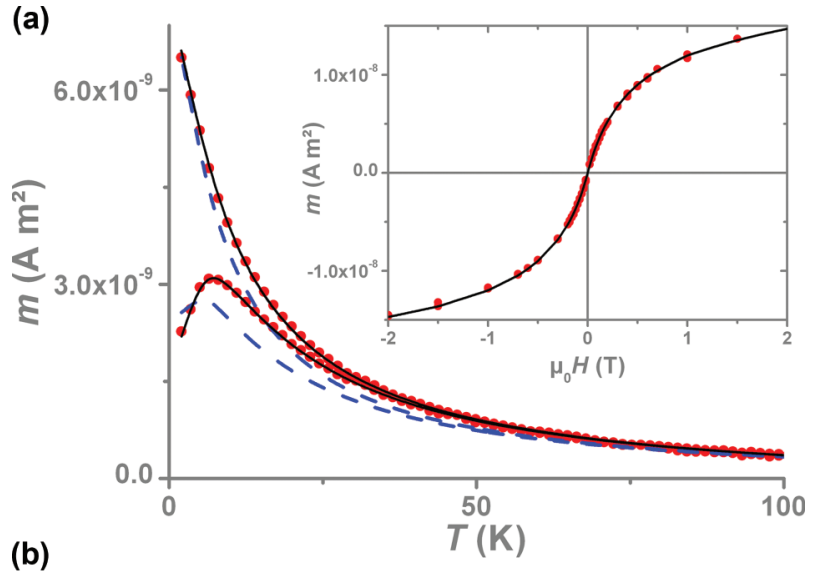

(b)

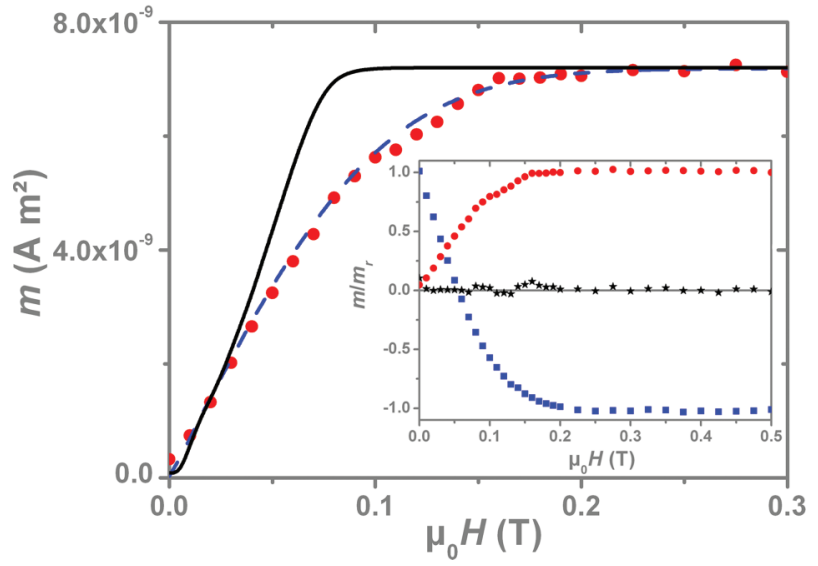

FIG. 1. (Color online) ZFC/FC curves (5 $\mathrm{mT}$ applied field), $300 \mathrm{~K}$ superparamagnetic loop (a) and IRM curve at $2 \mathrm{~K}$ (b) for the Co nanoparticle sample. The experimental measurements (dots) are compared to the theoretical curves using a uniaxial anisotropy with a single $K_{\text {eff }}$ (solid line, corresponding to the "triple fit" result) or a Gaussian $K_{\text {eff }}$ distribution (dashed line, corresponding to the IRM best fit, with the same particle size distribution). The experimental $\Delta m$ curve (stars), deduced from the IRM (dots) and DcD (squares) curves, is shown in the inset in (b).

$(\Delta m<0)$ interactions between nanomagnets, ${ }^{30}$ which allows us to safely apply the theoretical framework exposed above.

In order to determine the Co clusters magnetic properties, we first use the triple fit procedure to determine the magnetic size distribution and the effective anisotropy constant. ${ }^{15}$ At this point, a fit is possible with a uniaxial anisotropy and a single $K_{\text {eff }}$ of $95 \pm 15 \mathrm{~kJ} \mathrm{~m}^{-3}$, a median diameter of $2.0 \pm 0.2 \mathrm{~nm}$, and a dispersion parameter of $0.35 \pm 0.05$ [see Fig. 1(a)]. We then try to reproduce the IRM measurement using these parameters: As shown in Fig. 1(b), the simulated curve is however in complete disagreement with the experimental data. The observed lower slope of the IRM curve suggests a broader switching field distribution, which can be modeled by the use of a $K_{\text {eff }}$ distribution which can reflect the different shapes/facetting of the particles. It has indeed already been shown that the surface of a nanoparticle can significantly affect its anisotropy. ${ }^{34,40-42}$ As the measurements are performed on an assembly of nanoparticles, with different surfaces or shapes, this leads to a distribution of $K_{\text {eff }}$ in the sample.

We find that a Gaussian $K_{\text {eff }}$ distribution allows us to reproduce the experimental IRM curve [see Fig. 1(b)]. However, the
$\mathrm{ZFC} / \mathrm{FC}$ curves and in particular the merging temperature are known to be very sensitive to the broadness of the switching energy barrier distribution, and such an anisotropy constant distribution appears to be incompatible with the ZFC/FC measurements [see Fig. 1(a)]. Concerning the $m(H)$ at $300 \mathrm{~K}$ the simulated curve (with Langevin functions) does not change because there is no influence of the anisotropy constant $K_{\text {eff }}$ in this regime.

This discrepancy can be understood by the different reversal process involved in low-field susceptibility measurements (thermal switching) and for the IRM (field assisted switching). To go further, note that a biaxial anisotropy also leads to a broadening of the switching field distribution, ${ }^{18}$ as compared to the uniaxial case, but will not modify the distribution of blocking temperature. ${ }^{43}$ The consequence is that the shape of ZFC/FC curves is sensitive to the $\left|K_{1}\right|$ term (lowest energy barrier) while that of IRM curves reflects both $\left|K_{1}\right|$ and $K_{2}$ contributions. The idea is then to use at the same time a biaxial anisotropy (by fixing a $K_{2} /\left|K_{1}\right|$ ratio for simplicity) and an anisotropy constant distribution to simultaneously reproduce the entire $\operatorname{IRM}(H)$ at $2 \mathrm{~K}, \mathrm{ZFC} / \mathrm{FC}$ curves and $m(H)$ at $300 \mathrm{~K}$. These curves involve different physical processes, which we want to successfully describe with a unique set of parameters. The results and the fitting parameters are displayed in Fig. 2(b). They reveal the presence of a significant biaxial anisotropy term $\left(K_{2} \simeq\left|K_{1}\right|\right)$ as well as an anisotropy constant distribution (relative standard deviation around 35\%) which appears to be necessary to reproduce the experimental curves. A simulated IRM with the same parameters and $K_{2}=0$ is also presented, to emphasize the importance of the biaxial term. In size selected CoPt alloy clusters (relative size dispersion around 8\%), a large anisotropy constant dispersion has been revealed using only a simultaneous fit of the ZFC/FC curves and $m(H)$ at $300 \mathrm{~K}^{44}$ The ZFC/FC curves are very sensitive to the factor $K D^{3}$, and then if the diameter distribution is well known and sharp enough it is possible to infer the anisotropy constant distribution directly from the triple fit. In the present study, the approach is more general because there is no need to have a very narrow size distribution: The $K_{\text {eff }}$ dispersion is revealed thanks to the IRM curve, and could not have been characterized using only $\mathrm{ZFC} / \mathrm{FC}$ curves. Let us emphasize that the reason why IRM curves allows us to determine more precisely the magnetic anisotropy of nanoparticles is that it is controlled by the ratio between the applied field and the switching field, the latter being related to the anisotropy field and not the anisotropy energy (in contrast to ZFC/FC curves where the key parameter is the ratio between the thermal energy and the anisotropy energy $K_{1} V$ ).

In order to validate the results obtained on this Co clusters assembly by the combined analysis of the IRM and other magnetization curves, we have simulated the $m(H)$ hysteresis loop at $2 \mathrm{~K}$ with the same parameters, ${ }^{18}$ and we find an excellent agreement. ${ }^{45}$ Furthermore, a statistical $\mu$-SQUID study, which enables the determination of the switching field of each individual nanomagnet, has been performed for a hundred of particles [see Fig. 3(a)]. The deduced anisotropy field distribution is presented on Fig. 3(b) together with the one inferred from the SQUID measurements: ${ }^{46}$ The two distributions are in good agreement ${ }^{47}$ (median $H_{A}$ and dispersion). Being able to extract, from standard magnetometry measurements 
(a)

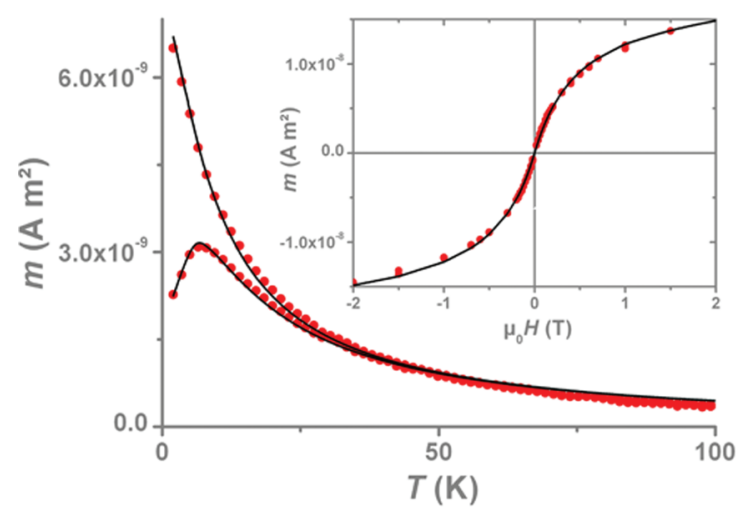

(b)

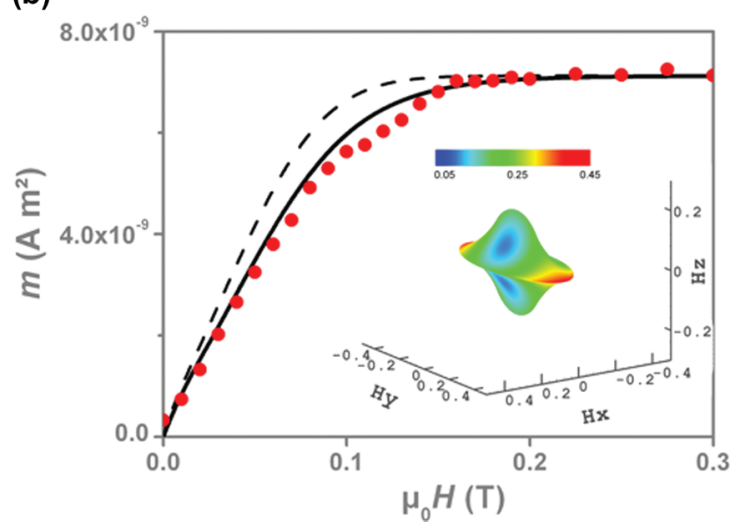

FIG. 2. (Color online) ZFC/FC curves (5 mT applied field), $300 \mathrm{~K}$ superparamagnetic loop (a) and IRM curve at $2 \mathrm{~K}$ (b) for the Co nanoparticle sample. The experimental measurements (dots) are compared to the theoretical curves (solid line, corresponding to the best global fit) using a biaxial anisotropy and an anisotropy constant distribution. The fits correspond to a median magnetic diameter $D_{m}=2.1 \mathrm{~nm}$, a dispersion parameter $w=0.31$, a mean effective anisotropy constant $K_{1}=115 \mathrm{~kJ} / \mathrm{m}^{3}$ with a standard deviation (anisotropy constant dispersion) of $\sigma_{K_{1}}=40 \mathrm{~kJ} / \mathrm{m}^{3}$, and a ratio $K_{2} /\left|K_{1}\right|=1.2$. The astroid (map of the switching field) corresponding to the median values is shown in the inset in (b). In dashed line the IRM curve simulated with $K_{2}=0$ is shown.

on a macroscopic nanoparticle assembly, the same type of information as from a cutting-edge unique experimental setup ( $\mu$-SQUID) is quite remarkable.

In conclusion, we have proposed a theoretical framework enabling the efficient simulation of IRM curves for realistic assemblies of magnetic particles with both a size and anisotropy constant distribution, and a biaxial anisotropy. Because they probe another type of switching process (field assisted switching), IRM curves are complementary ZFC/FC curves. The experimental IRM curve of Co clusters embedded in a carbon matrix has thus been analyzed, in combination with other magnetometry measurements, resulting in a powerful technique of magnetic properties characterization. In particular, we have been able to put into evidence an anisotropy constant dispersion among pure Co nanoparticles, confirmed by single particles measurements with a $\mu$-SQUID device. IRM curves then constitute a highly valuable way to probe
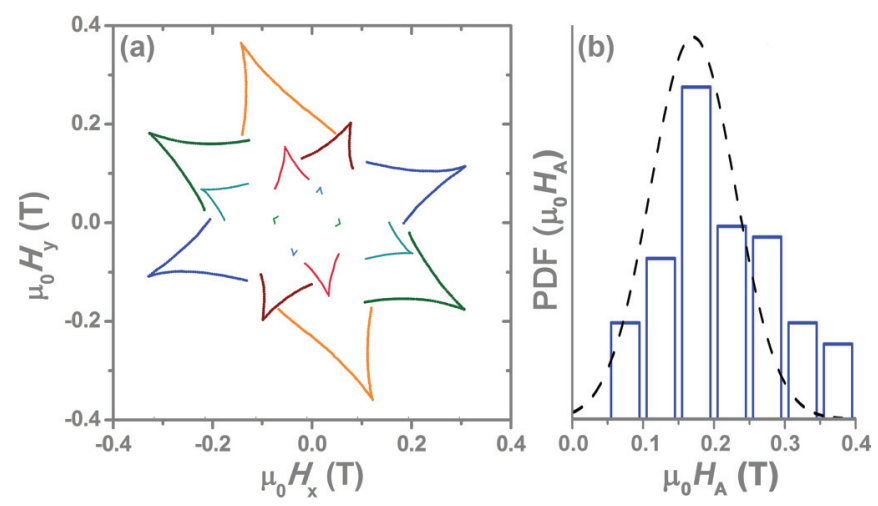

FIG. 3. (Color online) Examples of $\mu$-SQUID measurements (a) allowing us to determine the anisotropy field $H_{A}$ of individual Co nanoparticles, together with the distribution [histogram in (b) deduced from a hundred of analysis]. The $H_{A}$ probability distribution function (PDF) inferred from the SQUID magnetometry investigation on a Co cluster assembly is also shown (dashed line) for comparison. the intrinsic properties of nanomagnets of any kinds, and are much easier to model than hysteresis loops.

The authors acknowledge funding from the ANR DYSC research project, and technical support from the PLYRA and the CML platform.

\section{APPENDIX: ANALYTICAL EXPRESSION OF THE IRM IN THE STONER-WOHLFARTH CASE}

We consider the case of uniaxial particles, randomly oriented, at zero temperature (Stoner-Wohlfarth model). For a given orientation (angle $\theta$ ) between the easy axis and the applied magnetic field, it is well known that the switching field can be written as

$$
H_{\mathrm{sw}}^{0}(\theta)=H_{A}\left(\cos ^{2 / 3} \theta+\sin ^{2 / 3} \theta\right)^{-3 / 2},
$$

$H_{A}$ being the anisotropy field [i.e., $H_{A}=2 K_{\text {eff }} /\left(\mu_{0} M_{S}\right)$ ]. The switching field can also be written in a different way, using the switching angle $\theta_{0}$ (the angle between the particle magnetic moment and the applied field, at the moment when the switching energy barrier disappears) which is directly related to $\theta$ through the equation $\tan \theta_{0}=-\tan ^{1 / 3} \theta$. We obtain the following expression:

$$
H_{\mathrm{sw}}^{0}(\theta)=H_{A} \frac{\left(1-\tan ^{2} \theta_{0}+\tan ^{4} \theta_{0}\right)^{1 / 2}}{\left(1+\tan ^{2} \theta_{0}\right)^{2}} .
$$

It is then possible to determine, for an applied field $H \in\left[H_{A} / 2, H_{A}\right]$, the range of particles orientations which will switch, i.e., the interval $\left[\theta_{1}, \theta_{2}\right]$ where the inequality $H>$ $H_{\mathrm{sw}}^{0}(\theta)$ is verified. Since the switching field curve is symmetric with respect to $\theta=\pi / 4$ which is also the minimum [with $\left.H_{\mathrm{sw}}^{0}(\pi / 4)=H_{A} / 2\right]$, we have $\theta_{1}=\pi / 4-\delta$ and $\theta_{2}=\pi / 4+\delta$, where $\delta$ depends on $H$ (it goes from zero for $H=H_{A} / 2$ to $\pi / 4$ for $H=H_{A}$ ).

The IRM curve can now be expressed, taking into account the random orientation of the easy axes in the particle 
assembly, as

$$
\begin{aligned}
\operatorname{IRM}(H) & =2 \int_{\pi / 4-\delta}^{\pi / 4+\delta} N_{\text {tot }} \mu \cos \theta \frac{\sin \theta}{2} d \theta \\
& =\frac{N_{\text {tot }} \mu}{2} \int_{\pi / 4-\delta}^{\pi / 4+\delta} \sin (2 \theta) d \theta,
\end{aligned}
$$

where $\mu$ is the magnetic moment of a particle $\left(\mu=M_{S} V\right)$ and $N_{\text {tot }}$ the total number of particles. By simple integration and noting that $N_{\text {tot }} \mu / 2=m_{R}$, we finally have

$$
\operatorname{IRM}(H)=m_{R} \sin (2 \delta)=m_{R} \cos \left(2 \theta_{1}\right) .
$$

This shows that in order to $\operatorname{know} \operatorname{IRM}(H)$, all the problem relies on the determination of $\theta_{1}$ defined by $H_{\mathrm{sw}}^{0}\left(\theta_{1}\right)=H$ (and $\theta_{1}$ being the solution below $\left.\pi / 4\right)$. By introducing the reduced parameters $h=H / H_{A}$ and $x=\tan ^{2} \theta_{0}$, we then have to solve the equation:

$$
h=\frac{\left(1-x+x^{2}\right)^{1 / 2}}{1+x},
$$

*alexandre.tamion@univ-lyon1.fr

${ }^{1}$ M. Shliomis and V. Stepanov, J. Magn. Magn. Mater. 122, 176 (1993).

${ }^{2}$ S. U. Son, Y. Jang, J. Park, H. B. Na, H. M. Park, H. J. Yun, J. Lee, and T. Hyeon, J. Am. Chem. Soc. 126, 5026 (2004).

${ }^{3}$ Y. Chen, F. Yang, Y. Dai, W. Wang, and S. Chen, J. Phys. Chem. C 112, 1645 (2008).

${ }^{4}$ W.-R. Lee, M. G. Kim, J.-R. Choi, J.-I. Park, S. J. Ko, S. J. Oh, and J. Cheon, J. Am. Chem. Soc. 127, 16090 (2005).

${ }^{5}$ Z. Fan, M. Shelton, A. K. Singh, D. Senapati, S. A. Khan, and P. C. Ray, ACS nano 6, 1065 (2012).

${ }^{6}$ A. K. Gupta and M. Gupta, Biomaterials 26, 3995 (2005).

${ }^{7}$ T. A. Larson, J. Bankson, J. Aaron, and K. Sokolov, Nanotechnology 18, 325101 (2007).

${ }^{8}$ A.-H. Lu, E. L. Salabas, and F. Schüth, Angew. Chem. 46, 1222 (2007).

${ }^{9}$ R. Sharma and C. J. Chen, J. Nanopart. Res. 11, 671 (2008).

${ }^{10}$ C. Xu, K. Xu, H. Gu, X. Zhong, Z. Guo, R. Zheng, X. Zhang, and

B. Xu, J. Am. Chem. Soc. 126, 3392 (2004).

${ }^{11}$ D. L. Huber, Small 1, 482 (2005).

${ }^{12}$ D. M. Newman, M. L. Wears, M. Jollie, and D. Choo, Nanotechnology 18, 205301 (2007).

${ }^{13}$ S. Sun, Science 287, 1989 (2000).

${ }^{14}$ P. Gambardella, S. Rusponi, M. Veronese, S. S. Dhesi, C. Grazioli, A. Dallmeyer, I. Cabria, R. Zeller, P. H. Dederichs, K. Kern et al., Science 300, 1130 (2003).

${ }^{15}$ A. Tamion, M. Hillenkamp, F. Tournus, E. Bonet, and V. Dupuis, Appl. Phys. Lett. 95, 062503 (2009).

${ }^{16}$ A. Tamion, C. Raufast, M. Hillenkamp, E. Bonet, J. Jouanguy, B. Canut, E. Bernstein, O. Boisron, W. Wernsdorfer, and V. Dupuis, Phys. Rev. B 81, 144403 (2010).

${ }^{17}$ F. Tournus, A. Hillion, A. Tamion, and V. Dupuis, Phys. Rev. B 87, 174404 (2013).

${ }^{18}$ A. Tamion, E. Bonet, F. Tournus, C. Raufast, A. Hillion, O. Gaier, and V. Dupuis, Phys. Rev. B 85, 134430 (2012).

${ }^{19}$ R. W. Chantrell, D. Weller, T. J. Klemmer, S. Sun, and E. E. Fullerton, J. Appl. Phys. 91, 6866 (2002). which corresponds to the simple second order equation

$$
x^{2}\left(1-h^{2}\right)-x\left(1+2 h^{2}\right)+\left(1-h^{2}\right)=0 .
$$

The smallest solution is given by

$$
x_{1}=\frac{\left(1+2 h^{2}\right)-\sqrt{12 h^{2}-3}}{2\left(1-h^{2}\right)} .
$$

Finally, $\theta_{1}$ can be deduced from $x_{1}$ because we have $\theta_{1}=\arctan \left(x_{1}^{3 / 2}\right)$. Note however that a simple analytical expression, the one given in the paper, can be obtained for the IRM curve. We can indeed take advantage of the equality $x_{1}{ }^{3}=\tan ^{2} \theta_{1}$, by writing $\cos \left(2 \theta_{1}\right)=\cos ^{2} \theta_{1}-\sin ^{2} \theta_{1}$ and then use the trigonometric relations $\cos ^{2}=1 /\left(1+\tan ^{2}\right)$ and $\sin ^{2}=\tan ^{2} /\left(1+\tan ^{2}\right)$ to obtain

$$
\operatorname{IRM}(H)=m_{R} \frac{1-x_{1}{ }^{3}}{1+x_{1}{ }^{3}}
$$

for $H \in\left[H_{A} / 2, H_{A}\right]$.
${ }^{20}$ N. A. Usov and Y. B. Grebenshchikov, J. Appl. Phys. 106, 023917 (2009).

${ }^{21}$ X. Batlle, M. García del Muro, and A. Labarta, Phys. Rev. B 55, 6440 (1997).

${ }^{22}$ J. Garcia-Otero, M. Porto, and J. Rivas, J. Appl. Phys. 87, 7376 (2000).

${ }^{23}$ O. Hellwig, A. Berger, T. Thomson, E. Dobisz, Z. Z. Bandic, H. Yang, D. S. Kercher, and E. E. Fullerton, Appl. Phys. Lett. 90, 162516 (2007).

${ }^{24}$ S. Laureti, G. Varvaro, A. M. Testa, D. Fiorani, E. Agostinelli, G. Piccaluga, A. Musinu, A. Ardu, and D. Peddis, Nanotechnology 21, 315701 (2010).

${ }^{25}$ S. A. Majetich and M. Sachan, J. Phys. D: Appl. Phys. 39, R407 (2006).

${ }^{26}$ J. M. Martinez Huerta, J. De La Torre Medina, L. Piraux, and A. Encinas, J. Appl. Phys. 111, 083914 (2012).

${ }^{27}$ P. I. Mayo, K. O’Grady, P. E. Kelly, J. Cambridge, I. L. Sanders, T. Yogi, and R. W. Chantrell, J. Appl. Phys. 69, 4733 (1991).

${ }^{28}$ J. Rivas, A. Kazadi Mukenga Bantu, G. Zaragoza, M. Blanco, and M. López-Quintela, J. Magn. Magn. Mater. 249, 220 (2002).

${ }^{29}$ A. D. C. Viegas, J. Geshev, L. S. Dorneles, J. E. Schmidt, and M. Knobel, J. Appl. Phys. 82, 3047 (1997).

${ }^{30}$ O. Henkel, Phys. Status Solidi B 7, 919 (1964).

${ }^{31}$ S. W. Charles, R. Chandrasekhar, K. O'Grady, and M. Walker, IEEE Trans. Magn. 64, 5840 (1988).

${ }^{32}$ A. Stancu and C. Papusoi, IEEE Trans. Magn. 30, 2750 (1994).

${ }^{33}$ A. Thiaville, Phys. Rev. B 61, 12221 (2000).

${ }^{34}$ M. Jamet, W. Wernsdorfer, C. Thirion, D. Mailly, V. Dupuis, P. Mélinon, and A. Pérez, Phys. Rev. Lett. 86, 4676 (2001).

${ }^{35}$ A. Tamion, C. Raufast, E. Bonet, V. Dupuis, T. Fournier, T. Crozes, E. Bernstein, and W. Wernsdorfer, J. Magn. Magn. Mater. 322, 1315 (2010).

${ }^{36}$ M. Jamet, W. Wernsdorfer, C. Thirion, V. Dupuis, P. Mélinon, A. Pérez, and D. Mailly, Phys. Rev. B 69, 024401 (2004).

${ }^{37}$ A. Thiaville, J. Magn. Magn. Mater. 182, 5 (1998). 
${ }^{38}$ A. Perez, V. Dupuis, J. Tuaillon-Combes, L. Bardotti, B. Prevel, E. Bernstein, P. Mélinon, L. Favre, A. Hannour, and M. Jamet, Advanced Engineering Materials 7, 475 (2005).

${ }^{39}$ A. Perez et al., Inter. J. Nanotech. 7, 523 (2010).

${ }^{40}$ N. Pérez et al., Nanotechnology 19, 475704 (2008).

${ }^{41}$ D. A. Garanin and H. Kachkachi, Phys. Rev. Lett. 90, 065504 (2003).

${ }^{42}$ H. Kachkachi and E. Bonet, Phys. Rev. B 73, 224402 (2006).

${ }^{43}$ As discussed in Y. Kalmykov and B. Ouari [Phys. Rev. B 71, 094410 (2005)], in the low field limit, the leading exponential term of the relaxation time only depends on the lowest energy barrier (i.e., $K_{1}$ term). Therefore, there will be no detectable influence of the $K_{2}$ term on the blocking temperature.
${ }^{44}$ F. Tournus, N. Blanc, A. Tamion, M. Hillenkamp, and V. Dupuis, Phys. Rev. B 81, 220405 (2010).

${ }^{45}$ See Supplemental Material at http://link.aps.org/supplemental/ 10.1103/PhysRevB.88.094419 for the comparison between the experimental and simulated hysteresis loops at $2 \mathrm{~K}$.

${ }^{46}$ For the $\mu$-SQUID experiments, since the temperature is almost $0 \mathrm{~K}$ the switching field is independent of the particle size and the maximum field (apex of the astroid) directly gives the anisotropy field $H_{A}=2\left|K_{1}\right| /\left(\mu_{0} M_{S}\right)$. For the SQUID measurements, the distribution of $H_{A}$ is deduced from the $\left|K_{1}\right|$ distribution obtained by the best fit procedure of the magnetometry curves.

${ }^{47}$ Note that the $\mu$-SQUID technique does not allow ones to measure low anisotropy fields. 